\title{
Management bei Luftnot Akutmaßnahmen
}

Tim Wörth

Patienten mit schwerer Atemnot empfinden diese als zutiefst bedrohlich. Neben dem reinen „Lufthunger" quält sie vor allem die panische Angst davor, zu ersticken. Jetzt heißt es, die Situation rasch und korrekt zu erfassen, um schnell eine möglichst effektive Therapie einzuleiten. Gar nicht so einfach, denn: Dyspnoe ist therapeutisch oft schwerer angehbar als ein akutes Schmerzereignis. Die Überlagerung mit anderen Symptomen erschwert die Sache zusätzlich. Der folgende Artikel dient als kurzer klinischer Leitfaden. Er soll Orientierung in der angespannten Akutsituation geben und helfen, die häufigsten und potenziell lebensbedrohlichen Krankheitsbilder zu erkennen.

\section{Der erste Eindruck $\nabla$}

Lehrbücher sind gut, die Realität ist anders

Eine lehrbuchartige, systematische Abhandlung verschiedener Differenzialdiagnosen und deren Symptomen geht an der Realität der Akutsituation oft vorbei. Wichtiger erscheinen da der erste Eindruck und der klinische Blick: Sie erlauben eine rasche Einschätzung der Situation und eröffnen die Möglichkeit, potenzielle Ursachen als unwahrscheinlich zu erkennen.

Worauf sollte man achten? Leider treffen die typischen klinischen Bilder nicht immer zu. Insbesondere bei sehr alten Patienten ist die Bandbreite der Symptomatik groß. Als erstes sollte erfasst werden:

- Ist der Patient tachypnoisch?

- Bestehen eine Zyanose und/oder periphere Ödeme?

- Ist das Atemgeräusch normal, abgeschwächt oder aufgehoben?

- Besteht ein Nebengeräusch bei der Atmung? $\triangleright$ Inspiratorisch feuchte Rasselgeräusche oder $\triangleright$ exspiratorisches Giemen/Pfeifen/Brummen (sog. trockene Rasselgeräusche)?

Die Auskultation ist das schnellste und am leichtesten verfügbare Mittel der Diagnostik, das meist schon die Festlegung auf eine Ursache der Dyspnoe erlaubt.
Anamnese Sofern der Patient noch antworten kann, sollten Sie in einer kurzen Anamnese erfragen,

- wie rasch die Atemnot entstanden ist und

- ob es einen Auslöser gab.

- Auch Vorerkrankungen der Lunge oder des Herzens sollten kurz anamnestisch erfasst werden.

Beruhigen Sie den Patienten! An dieser Stelle kommt dem Arzt-Patienten-Kontakt besondere Bedeutung zu:

- Beruhigende Worte helfen manchmal Wunder,

- ein dem Patienten zugewandtes und strukturiertes Auftreten lindert die Angst.

- Es ist wichtig, ein ruhiges Umfeld zu schaffen, ggf. auch besorgte Angehörige vor die Tür zu schicken.

Der Patient erwartet vom Arzt in dieser Situation Hilfe. Diese müssen Sie nicht nur durch Taten, sondern auch verbal vermitteln.

\section{Weitergehende Diagnostik}

Abhängig vom Schweregrad Die weiteren Schritte der Diagnostik müssen im Einzelfall der Ausprägung der Dyspnoe und dem Grad der vitalen Bedrohung des Patienten angepasst werden. Prinzipiell sollte, wenn immer möglich,

- eine pulsoxymetrische Messung der Sauerstoffsättigung erfolgen und

- der Patient mittels EKG- und Blutdruckmessung überwacht werden.

- Ferner ist ein 12-Kanal-EKG unerlässlich.

Beides ist meist ohne Zeitverzögerung möglich.

- Sehr aufschlussreich ist die arterielle Blutgasanalyse, um Kenntnis über das Ausmaß der etwaigen Hypoxie und Hyperkapnie zu erlangen.

- Letztendlich ist ein Röntgen-Thorax, im Stehen und in 2 Ebenen, eine wichtige diagnostische Maßnahme. Sie sollte, sofern möglich, unbedingt angestrebt werden. 
Manchmal wird aufgrund des Allgemeinzustands des Patienten nur eine Aufnahme im Liegen möglich sein, manchmal wird man sie in der kritischen Notfallsituation erst nach der Intubation anfertigen können.

\section{Was tun?}

Ursache klassifizieren Eine einfache Klassifikation zeigt $\bullet$ Abb. 1. Als „erster diagnostischer Angriff“ genügt es, den Patienten abzuhören:

- „Brodelt“ der Patient, besteht eine alveoläre Ansammlung von Flüssigkeit - es sind inspiratorisch feuchte Rasselgeräusche auskultierbar.

- „Giemt“ oder „pfeift“ der Patient beim Ausatmen, liegt eine bronchiale Obstruktion vor.

- Übrig bleibt das heterogene Feld anderer Ursachen.

Cave Die Therapiestrategien bei alveolär und bronchial verursachter Dyspnoe unterscheiden sich grundlegend! Gemeinsam ist ihnen nur die Gabe von Sauerstoff als wichtigste Erstmaßnahme.

- Diese sollte unbedingt über eine Mund-NasenMaske mit Reservoir erfolgen, da nur so hohe inspiratorische Sauerstoff-Fraktionen erreicht werden.

- Eine Nasensonde oder Sauerstoff-Brille ist in jedem Fall unzureichend.

\section{Bei alveolärem Ödem: Venen weiten}

Morphin ist ideal Der „brodelnde“ Patient profitiert von Volumenentlastung: Am schnellsten hilft das „venöse Pooling“ durch Erweiterung der herznahen Venen. Ideal ist hier Morphin, das zusätzlich noch eine sedative Wirkung hat und somit das Symptom der Luftnot effektiv lindert.

- Fraktioniert in 2-mg-Boli das Morphin unter Kontrolle von Blutdruck und Vigilanz langsam aufdosieren.

- Nach insgesamt $10 \mathrm{mg}$ Morphin sollte zunächst abgewartet werden, da die volle Wirkung frühestens nach ca. 15 min zu erwarten ist.

Die Angst vor der Atemdepression ist bei vorsichtiger und fraktionierter Dosierung meist unbegründet: Der nach Luft schnappende, agitierte Patient hat einen solch hohen sympathikotonen Antrieb, dass Morphin in normaler Dosierung keinen Atemstillstand verursacht.

Nitrate wirken schneller Das venöse Pooling kann noch rascher durch die Gabe von Nitraten (als Spray s.1.) erreicht werden. Hierbei ist natürlich die Blutdruckkontrolle entscheidend, um bedrohliche Hypotonien zu verhindern.

- Als Erstgabe empfehlen sich 1-2 Hub Nitrospray - abhängig von Blutdruck und Wirkung.
- Dabei die Wirksamkeit für einige Minuten beobachten.

- Ggf. kann die Gabe wiederholt werden.

Mechanisches Absenken der Vorlast Ebenfalls wirksam, aber in der Praxis selten angewendet, ist der „unblutige Aderlass“. Hierbei wird mittels Blutdruckmanschetten an allen 4 Extremitäten der venöse Rückstrom gedrosselt. Um die periphere Perfusion nicht zu stark zu limitieren, werden abwechselnd immer nur 3 Extremitäten für 10 min gestaut. Der Manschettendruck sollte nur eine venöse Stauung bewirken.

Diuretika sind nur bedingt geeignet Selbstverständlich kommen auch Diuretika, v.a. das Schleifendiuretikum Furosemid in einer Erstdosis von 40-80 mg i.v., zum Einsatz. Ihre Wirkung darf man aber nicht zu schnell erwarten: Insbesondere Furosemid muss erst im Primärharn filtriert werden, bevor es von luminaler Seite in der Henle-Schleife wirken kann - das dauert einige Zeit.

- Bekanntermaßen niereninsuffiziente Patienten benötigen oft eine wesentlich höhere Dosis.

- Zusätzlich ist in ihrem Fall oft die Pufferung zum Ausgleich einer metabolischen Azidose nötig, um überhaupt eine Diurese zu erreichen.

- Vorsicht ist dann bei der Anwendung von Bikarbonat geboten: Das bei der Pufferung anfallende $\mathrm{CO}_{2}$ kann bei insuffizienter Atmung möglicherweise nicht mehr abgeatmet werden!

Ist der Patient anurisch, sind Diuretika sinnlos.

Der schnell wirksame therapeutische Mechanismus beim alveolären Ödem ist das venöse Pooling durch Morphin oder Nitrate.

Verwendung von Atemmasken Eine apparative Option ist die nicht-invasive Maskenbeatmung mittels CPAP (continuous positive airway pressure). Der Patient muss hierfür allerdings bei ausreichender Vigilanz und kooperationsfähig sein. Die engsitzende Maske wird darüber hinaus leider oft als sehr beengend empfunden.

- Wichtig: Klären Sie den Patienten über die Maßnahme auf und

- setzen Sie die Maske zunächst mit der Hand auf das Gesicht des Patienten.

- Unter beruhigenden Worten lässt sich meist die Toleranz der Maske erreichen.

So lässt sich Luftnot klassifizieren

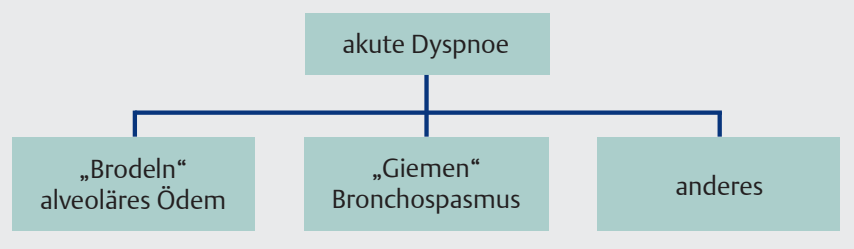

Abb. 1 Einfaches Schema zur Klassifizierung der Ursachen von Luftnot. Durch das Abhören des Patienten lassen sich die dafür nötigen Informationen gewinnen. 
- Erst wenn dies funktioniert, sollte die Maske mit den Bändern am Kopf des Patienten befestigt werden.

Intubation Ist die Vigilanz so stark eingeschränkt, dass der Patient nicht mehr kooperationsfähig ist, dann ist auch ein Masken-CPAP nicht mehr möglich. Hier bleibt nur die endotracheale Intubation. Die Entscheidung zur Intubation stellt oft eine Hemmschwelle dar, insbesondere für Nicht-Anästhesisten. „Soll man oder soll man nicht?“ ist dann die angstbesetzte Frage.

Praxistipp Meist ist die Indikation zur Intubation gegeben, sobald man daran denkt.

So wird die Intubation deutlich leichter:

- Die Narkose erst beginnen, wenn das Equipment vollständig und griffbereit ist.

- Kapnometrie und leistungsfähige Absaugung parat halten.

- Eine Larynxmaske und/oder einen Larynxtubus für den Fall des unerwartet schwierigen Atemweges bereit halten.

- Richtige Lagerung des Patienten (für RSI-Einleitung erhöhter Oberkörper).

- Richtige Lagerung des Kopfes in „SchnüffelStellung“ (d.h. ein Polster unter den Hinterkopf legen oder Intubationskissen verwenden).

- Wenn irgend möglich, gute Präoxygenation mit $100 \%$ Sauerstoff über die engsitzende Maske für einige Minuten.

- Ausreichend tiefe Narkose.

- Im Zweifelsfall erfahrene Hilfe hinzuholen.

\section{Bei Bronchospasmus: Bronchien weiten} $\nabla$

Ausatmen erleichtern per Inhalation Der „giemende“ Patient imponiert durch ein verlängertes Exspirium - verursacht durch die Engstellung der unteren Atemwege. Sein Problem ist das Ausatmen, was sich somit fundamental vom alveolären Ödem bei Pneumonie oder Lungenödem unterscheidet. Der therapeutische Ansatz ist die Bronchodilatation.

- Schnell wirksam sind hierbei $\beta_{2}$-Sympathomimetika, wie Reproterol oder Salbutamol.

- Sofern möglich, sollte die Gabe inhalatorisch erfolgen (z. B. 5 Hübe Salbutamol als Erstgabe). Die Applikation der handelsüblichen Sprays gelingt in der Notfallsituation oft nur ineffektiv.

- Besser ist dann eine Inhalationsmaske, in deren Vernebler die Medikamente in flüssiger Form eingegeben werden und die über einen hohen Sauerstofffluss ein sehr feines Aerosol erzeugt.

Alternative Adrenalin Nicht zu vergessen ist allerdings der stärkste Bronchodilatator - Adrenalin! Eine sehr gut wirksame Alternative ist somit die inhalative Gabe von Adrenalin
- in einer Verdünnung von $1 \mathrm{mg}$ Adrenalin auf 2-4 ml NaCl-Lösung 0,9\%.

$\mathrm{Zu}$ beobachten sind in jedem Fall Herzfrequenz und Herzrhythmus, was ein lückenloses Monitoring des Patienten nötig macht.

Andere Applikationsformen Reproterol und Salbutamol können auch intravenös, Terbutalin auch subkutan verabreicht werden. Hier sollte die Gabe fraktioniert unter o.g. Monitoring erfolgen.

Kortikosteroide wirken langsam Zuletzt kommen Glukokortikoide (Prednisolon oder Methylprednisolon) zum Einsatz. Ihre Wirkung tritt allerdings erst nach frühestens 15-30 min durch ein Abschwellen der Bronchialschleimhaut ein.

Maskenbeatmung Auch bei einem Bronchospasmus lässt sich das Masken-CPAP erfolgreich anwenden, einerseits zur Linderung des Symptoms „Dyspnoe“, andererseits, um wirkungsvoll einer Hyperkapnie zu begegnen.

Die Inhalation von Bronchodilatatoren wirkt dem Bronchospasmus am schnellsten entgegen. Adrenalin verdünnt ist hier hervorragend wirksam.

Besonderheiten bei COPD Patienten mit chronisch obstruktiver Lungenerkrankung und daraus resultierender chronischer Hyperkapnie können auf die Sauerstoffgabe mit einem Verlust des Atemantriebs reagieren. Aufgrund des stets erhöhten arteriellen $\mathrm{CO}_{2}$-Partialdrucks erfolgt die Regulation des Atemantriebs über den chronisch erniedrigten $\mathrm{O}_{2}$-Partialdruck. Hier sind Vigilanz und Atemfrequenz das entscheidende klinische Merkmal.

Mehrere Ursachen gleichzeitig Vor allem bei multimorbiden und betagten Patienten kommt es oft zu Mischformen der beiden bisher genannten Krankheitsbilder „alveoläres Ödem“ und „Bronchospasmus“.

Bei Mischformen orientiert man sich zunächst am führenden Symptom. Es kann im Zweifelsfall aber auch zweckdienlich sein, quasi zweigleisig beide Therapiestrategien zu verfolgen.

\section{Bei anderen, potenziell lebensbedrohlichen Ursachen}

$\nabla$

Lungenembolie Die Verstopfung der Lungenarterien kann sich vielgestaltig äußern: Vom beschwerdefreien bis zum reanimationspflichtigen Patienten sind alle klinischen Bilder denkbar. Die häufigsten Symptome sind

- Dyspnoe,

- Tachykardie und 
- Thoraxschmerz.

Entscheidend ist es (so banal es sich anhört), überhaupt an die Möglichkeit einer Lungenembolie zu denken und in einer kurzem Anamnese deren Risikofaktoren abzufragen. Dies sind z. B.:

- klinische Hinweise auf Thrombose

- kürzlich stattgehabte Operation

- Immobilisation durch Krankheit oder lange Flug- oder Busreise

- Exsikkose

- Trauma

- Thrombophilie

Die definitive Diagnostik erfolgt mittels Echokardiografie und Thorax-CT mit Kontrastmittelgabe.

- Bei hochgradigem Verdacht auf eine Lungenembolie und Fehlen von Kontraindikationen gegen eine Antikoagulation können 10000 IE unfraktioniertes Heparin i.v. gegeben werden.

- Ansonsten erfolgt vor Diagnose-Sicherung zunächst nur die Sauerstoffgabe und die symptomatische Therapie.

- Bei hochgradigem Verdacht und Reanimation kann die Ultima-ratio-Lyse erfolgen.

Spontan-Pneumothorax Das führende Symptom ist meist ein

- stechender, plötzlich auftretender Thoraxschmerz, verbunden mit Dyspnoe.

- Das Atemgeräusch ist einseitig meist deutlich abgeschwächt oder gänzlich aufgehoben.

Typischerweise wird der junge, asthenische Patient von einem Spontan-Pneumothorax ereilt.

- Die Erstmaßnahme ist immer die Sauerstoffgabe.

- Die Diagnosesicherung erfolgt mittels RöntgenThorax in 2 Ebenen, idealerweise in Exspiration!

- Je nach Ausprägung kann die Therapie konservativ oder über eine Thoraxdrainage erfolgen.

Spannungs-Pneumothorax Gefürchtet ist der (seltene) Spannungs-Pneumothorax, der über einen Ventilmechanismus zu einer massiven Druckerhöhung im Pleuraspalt führt und die herznahen Venen komprimiert. Symptome sind neben den o.g.

- zusätzlich Tachykardie,

- Hypotonie und zuletzt

- Schock,

wobei sich das klinische Bild des Patienten rasant verschlechtert.

- In diesem Fall ist die unmittelbare Punktion der Pleurahöhle nötig, um eine Druckentlastung zu erreichen.

Aspiration An die Aspiration muss vor allem bei Kindern und bei betagten Menschen gedacht werden. Eine (teilweise) Verlegung der Atemwege im Bereich des Kehlkopfes oder der Trachea führt zu einem inspiratorischen Stridor (nicht zu verwechseln mit dem exspiratorischen Giemen beim Bronchospasmus).
- Bei milder Symptomatik sollte der Versuch des Absaugens oral oder - meist einfacher - nasal erfolgen, wobei man während eines Hustenstoßes den Absaugkatheter oft bis in die Trachea vorschieben kann.

- Bei bedrohlichem Zustand bleiben nur Narkose und Intubation. Hier kann laryngoskopisch die Stimmritze inspiziert und ggf. ein Fremdkörper mittels Magill-Zange entfernt werden. Anschließend sind die Bronchoskopie und ein Röntgen-Thorax unumgänglich.

Fazit Die akute Dyspnoe muss rasch und wirkungsvoll behandelt werden. Eine schnelle (Verdachts-)Diagnose ist essenziell. Die Erstmaßnahme ist immer die Sauerstoffgabe, bevorzugt via Gesichtsmaske. Eine erste Festlegung mittels Auskultation ermöglicht die Differenzierung zwischen alveolärem Ödem und Bronchospasmus oder anderen Ursachen. Die Therapie zielt im ersten Fall auf eine Senkung der Vorlast und Reduktion des Flüssigkeitsvolumens. Im zweiten Fall setzt die Therapie bei Bronchodilatation und Abschwellen der Bronchialschleimhaut an. Die anderen (und selteneren) Ursachen erfordern eine differenziertere Diagnostik und Therapie.

\section{Checkliste}

- Gib dem Patienten Sauerstoff über eine MundNasen-Maske mit Reservoir.

- Differenziere die Ursache rasch mittels kurzer Anamnese und Auskultation.

Sorge durch ein adäquates Monitoring für Sicherheit des Patienten.

- Strebe 12-Kanal-EKG, arterielle Blutgasanalyse und Röntgen-Thorax an.

- Der schnelle Mechanismus beim alveolären Ödem ist das venöse Pooling durch Nitrate und Morphin.

- Der schnelle Mechanismus beim Bronchospasmus ist die inhalatorische Therapie, ggf. mit Adrenalin.

- Denke an seltenere, aber gefährliche Ursachen wie Pneumothorax, Lungenembolie oder Aspiration.

- Denke an die Möglichkeit der nicht-invasiven Maskenbeatmung.

Zögere nicht mit der Intubation, wenn Du sie als indiziert ansiehst.

Beitrag online zu finden unter http://dx.doi. org/10.1055/s-s-0031-1272354

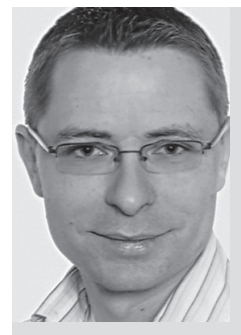

Dr. med. Tim Wörth ist Facharzt für Innere Medizin an der Klinik für Anästhesie, Intensivmedizin, Schmerztherapie und Notfallmedizin am Klinikum Ludwigsburg. E-Mail:

Tim.Woerth@kliniken-lb.de

\section{Interessenkonflikt}

Der Autor erklärt, dass keine wirtschaftlichen oder persönlichen Verbindungen bestehen. 